\title{
Évaluation de la résistance à la salinité au stade jeune plant de quelques cultivars de piment (Capsicum spp.) du Benin
}

Julien Koffi Kpinkoun1, Séraphin Ahissou Zanklan1, Françoise Assogba Komlan², Armel C. G. Mensah2, David Montcho ${ }^{3}$, Eliane Kinsou ${ }^{1}$ et Christophe Bernard Gandonou ${ }^{1 *}$

1 Unité de Recherche sur l'Adaptation des Plantes aux Stress Abiotiques, les Métabolites Secondaires et l'Amélioration des Productions Végétales, Laboratoire de Physiologie Végétale et d'Etude des Stress Environnementaux, Faculté des Sciences et Techniques (FAST/UAC), 01BP526, Tri Postal, Cotonou, République du Bénin.

${ }^{2}$ Centre de Recherches Agricoles Plantes Pérennes (CRA-PP), Pobè, Institut National des Recherches Agricoles du Bénin (INRAB), Abomey-Calavi, Bénin

${ }^{3}$ Ecole de Gestion de la Production Végétale et Semencière, Université Nationale d'Agriculture (Kétou), République du Bénin.

Auteur pour correspondance : Courriel : ganchrist@hotmail.com, Tél. : (00229) 97396978

Original submitted in on 9th October 2018. Published online at www.m.elewa.org on $31^{\text {st }}$ January 2019 https://dx.doi.org/10.4314/jab.v133i1.8

\section{RÉSUMÉ}

Objectifs : La salinité est un facteur abiotique qui influe négativement sur la physiologie des plantes. Cette étude vise à évaluer l'effet de différentes concentrations de $\mathrm{NaCl}$ (Chlorure de sodium) sur la croissance des plants de cinq cultivars (Adologbo, Gbatakin, TPS 0251, Gbatakin d'Agbédranfo et Démon) de piment (Capsicum spp) produits au Bénin afin de déterminer leur niveau relatif de résistance au stress salin.

Méthodologie et résultats : Cinq concentrations de $\mathrm{NaCl}$ comprises entre 0 et $120 \mathrm{mM}$ ont été appliquées pendant deux semaines sur les plants de piment âgés de trois semaines dans un dispositif complétement randomisé avec quatre répétitions. Les résultats indiquent que l'effet du sel s'est traduit par une réduction des différents paramètres de croissance étudiés avec une différence significative entre les cultivars. Les réductions observées ont été moins accentuées chez le cultivar Gbatakin alors qu'elles ont été plus accentuées chez TPS 0251 et Adologbo. L'indice de tolérance au sel le plus élevé a été observé chez le cultivar Gbatakin et le plus faible chez Adologbo et TPS 0251.

Conclusion et applications des résultats : Le stress salin réduit la croissance des cinq cultivars de piment évalués quel que soit le paramètre de croissance pris en compte. Le cultivar Gbatakin maintient une meilleure croissance en présence du $\mathrm{NaCl}$ avec l'indice de tolérance au sel le plus élevé et apparaît de ce fait comme le plus résistant des cinq cultivars testés tandis que les cultivars Adologbo et TPS 0251 qui présentent les croissances les plus faibles en présence de $\mathrm{NaCl}$ avec les indices de tolérance au sel les plus faibles sont les plus sensibles. A ce stade de nos résultats, le cultivar Gbatakin peut être conseillé aux producteurs des zones côtières ou affectées par la salinité tandis que les producteurs des autres zones peuvent produire tous les cultivars.

Mots clés : Capsicum spp, croissance, $\mathrm{NaCl}$, indice de tolérance au sel. 


\title{
Evaluation of the resistance to salinity of some chili pepper (Capsicum spp.) cultivars produced in Benin
}

\begin{abstract}
Objectives: Salinity is an abiotic factor that negatively influences the physiology of plants. This study aims to evaluate the effect of different concentrations of Sodium chloride $(\mathrm{NaCl})$ on plant growth of five cultivars of chili (Adologbo, Gbatakin, TPS 0251 Gbatakin d'Agbédranfo, and Démon) grown in Benin to determine their relative level of resistance to salt stress.

Methodology and results: Five $\mathrm{NaCl}$ concentrations from 0 to $120 \mathrm{mM}$ were applied for two weeks on the three weeks old plants in a fully randomized four-repeat device. The results indicate)d that the salt effect resulted in a reduction of the different growth parameters (like plant height, shoot fresh and dry masses, leaf number, root length, root fresh and dry masses) studied with a significant difference between cultivars. The reductions observed were less pronounced in cultivar Gbatakin whereas they were more pronounced in TPS 0251 and Adologbo. The highest salt tolerance index was observed in Gbatakin and the lowest in Adologbo and TPS 0251.

Conclusion and applications of the results: Salt stress reduced the growth of the five chilli cultivars evaluated regardless of the growth parameter taken into account. The cultivar Gbatakin maintained better growth in the presence of $\mathrm{NaCl}$ with the highest salt tolerance index and thus appears to be the most resistant of the five cultivars tested, while Adologbo and TPS 0251 which showed the lowest growth under salt stress with the lowest salt tolerance indexes are the most sensitive. From these results, cultivar Gbatakin can be recommended to growers in coastal or salinity-affected areas while producers in other areas can produce all chilli cultivars.
\end{abstract}

Keywords: Capsicum spp., plant growth, $\mathrm{NaCl}$, salt tolerance index.

\section{Abréviations : \\ $\mathrm{CRH}$ : Croissance relative en hauteur des plants \\ CRLR : Croissance relative en longueur des racines \\ CRNF : Croissance relative du nombre de feuilles \\ CRSF : Croissance relative de la surface foliaire}

MFPA : Masse de matière fraîche de la partie aérienne MFPR : Masse de matière fraîche de la partie racinaire MSPA : Masse de matière sèche de la partie aérienne MSPR : Masse de matière sèche de la partie racinaire

\section{INTRODUCTION}

La salinité est l'une des contraintes environnementales les plus importantes qui limitent la productivité des plantes particulièrement dans les climats aride et semi-aride (Hussain et al., 2009). On estime à 800 millions d'hectares la surface cultivable touchée par la salinité du sol à travers le monde (Munns \& Tester, 2008). Cette surface ne cesse d'augmenter en raison des mauvaises pratiques agricoles et l'augmentation du niveau de la mer (Munns, 2005). L'excès d'ions salins dans la solution du sol génère à la fois une pression osmotique élevée et une accumulation d'ions devenant toxiques dans les feuilles notamment le $\mathrm{Na}^{+}$, ce qui a comme conséquence une réduction de la croissance de la production des cultures due à une perturbation de plusieurs processus physiologiques des plantes (Munns, 2002). La croissance des plantes est compromise par la salinité à tous les stades de développement, mais la sensibilité varie beaucoup à différents stades (Caravajal et al., 1998 ; Akram et al., 2002 ; Akinci et al., 2004). La production végétale dans les zones salines dépend en grande partie de la réussite de la germination, de la levée, de la phase de croissance et de l'efficacité de la phase reproductrice (Bhattacharjee, 2008). Bien que presque toutes les plantes cultivées soient des espèces glycophytes, leurs réponses globales à l'augmentation de la dose de $\mathrm{NaCl}$ semblent être spécifiques à l'espèce (Levitt 1980 ; Lutts et al., 1995 ; Karikalan, 1999 ; Lakra, 2006 ; Chukwu \& Okpe, 2006 ; Gandonou et al., 2012 ). De plus, au 
sein d'une même espèce donnée, une variation substantielle de la sensibilité au sel peut apparaître chez les cultivars (Lutts et al., 1995 ; Wei et al., 2003; Gandonou et al., 2012). Le piment (Capsicum spp.) fait partie des cultures maraîchères les plus importantes au Bénin et mobilise à lui seul plus de $1 \%$ des superficies consacrées au niveau national pour la production des cultures vivrières (Assogba-Komlan et al., 2009). La culture du piment est pratiquée dans toutes les régions du Bénin notamment sur les plateaux, dans les plaines alluviales, dans les vallées, dans les bas-fonds et sur le littoral avec quatre systèmes de cultures à savoir : le système pluvial, la culture de décrue, la culture de bas-fond et le système irrigué (PNUD, 2015). Les départements du Sud-Bénin fournissent environ $70 \%$ de la production nationale (Assogba-Komlan et al., 2009). Le piment joue un rôle important dans l'alimentation des populations et contribue à la

\section{MATERIEL ET MÉTHODES}

Matériel végétal : Cinq (05) cultivars de piment dont quatre cultivars locaux (Adologbo, Gbatakin, TPS 0251 et Gbatakin d'Agbédranfo), et une variété importée (Démon) ont été utilisés. Les semences des quatre cultivars locaux ont été fournis par le sous-programme 'Cultures Maraîchères' du Programme 'Plantes Pérennes' de I'Institut National des Recherches Agricoles du Bénin (INRAB), tandis que les semences de la variété importée ont été achetées auprès de la société 'Bénin Semences'.

\section{Méthodologie}

Conditions expérimentales : L'expérience a été réalisée dans une serre à l'Institut National des Recherches Agricoles du Bénin (INRAB)/ (AbomeyCalavi, République du Bénin) de février à avril 2017. Les plantes ont été cultivées à une température de 26 ${ }^{\circ} \mathrm{C} / 22{ }^{\circ} \mathrm{C}$ jour / nuit avec de la lumière naturelle et une humidité relative de $55 \%$. Les graines ont été mises en germination dans des bacs remplis de terreau pendant deux semaines. Les jeunes plants ont été ensuite transférés dans des petits pots de $5,8 \mathrm{~cm}$ de diamètre et de $6 \mathrm{~cm}$ de hauteur contenant un mélange de terreau et de sable 50:50 (une plante/pot) et cultivés pendant une semaine avant l'application du stress. Les plants des cinq cultivars ont été soumis à un stress salin dans des grands pots de terre de $11,3 \mathrm{~cm}$ de diamètre et de $14 \mathrm{~cm}$ de hauteur remplis avec $3 \mathrm{~kg}$ du même mélange. prévention des maladies dues aux carences en micronutriments (PSRSA, 2011). Une bonne partie de la zone de production du piment est située en zone côtières où la salinité du sol et de l'eau d'irrigation constitue de véritables problèmes entravant la production des cultures. Les producteurs maraîchers du Sud-Bénin reconnaissent la salinité des eaux d'irrigation et les embruns marins comme un problème majeur pour leurs activités (Orobiyi et al., 2013; Déguénon, 2018). Cependant, en dépit d'une quantité considérable de travaux sur les réponses des plantes au stress salin, il n'y a pratiquement pas de travaux sur la résistance au sel des cultivars de piment cultivés au Bénin. La présente étude vise à combler cette insuffisance à travers l'évaluation des effets du stress $\mathrm{NaCl}$ sur la croissance des jeunes plants de cinq cultivars de Capsicum spp. cultivés au Bénin afin de déterminer leur niveau relatif de résistance à la salinité.

Les traitements ont consisté à l'arrosage des plantes tous les deux jours avec $100 \mathrm{ml} /$ pot de solution saline de $0 ; 30 ; 60 ; 90$ ou $120 \mathrm{mM}$ de $\mathrm{NaCl}$ correspondant respectivement à des conductivités électriques de 0 ; 1,$91 ; 4,81 ; 8,39 ; 12.85$ dS.m-1 déterminées par un conductimètre (VWR, CO310).

Dispositif expérimental : Le dispositif expérimental est de type factoriel à deux facteurs en randomisation complète. Le premier facteur représente les cinq (05) traitements salins $(\mathrm{T} 0=0 \mathrm{mM} ; \mathrm{T} 1=30 \mathrm{mM} ; \mathrm{T} 2=60$ $\mathrm{mM}$; $\mathrm{T} 3=90 \mathrm{mM}$ et T4= $120 \mathrm{mM}$ de $\mathrm{NaCl}$ ) et le 2 ème facteur est représenté par les cinq (05) cultivars de piment (Adologbo, Gbatakin, Gbatakin d'Agbédranfo, TPS 0251 et Démon) avec quatre répétitions pour chaque cultivar.

Evaluation de l'expérimentation : La hauteur des plantes, la surface foliaire, le nombre de feuilles et la longueur de la racine ont été mesurées avant l'application du stress salin ( $\mathrm{X} 0)$; ils ont été mesurés à nouveau après 2 semaines de traitement (X1). La croissance relative en hauteur des plantes (CRHP), la croissance relative du nombre de feuilles (CRNF) et la croissance relative de la longueur des racines (CRLR) ont été déterminés suivant la formule: $(\mathrm{X} 1-\mathrm{X} 0)$ / X0. Les masses de matière fraîche de la partie aérienne et des racines ont été déterminées par pesée à la fin des traitements (après deux semaines). Les échantillons de 

quelques cultivars de piment (Capsicum spp.) du Benin

chaque partie ont ensuite été transférés dans une étuve à $80^{\circ} \mathrm{C}$ pendant 72 heures pour déterminer la masse sèche. L'indice de tolérance au sel (ITS) est calculé pour chaque cultivar et pour chaque paramètre de croissance pris en compte selon la formule adaptée de celle de Tabatabaei et al. (2012).

\section{RESULTATS}

Comportement global des cinq cultivars de piment vis-à-vis du stress salin : L'analyse de variance à deux facteurs a révélé un effet significatif du stress salin pour l'ensemble des paramètres de croissance pris en compte et une différence significative entre les cultivars à l'exception du nombre de feuilles (Tableau
Analyses statistiques : L'analyse des effets des cultivars et de l'intensité du stress a été basée sur l'analyse de la variance (ANOVA) à un ou deux facteurs selon le cas. Les moyennes ont été comparées en utilisant le test de Student, Newman et Keuls. Les analyses statistiques ont été réalisées en utilisant le logiciel “JMP Pro 12" (SAS Institute, 2015).

1). Cependant, l'interaction entre les deux facteurs n'est significative pour aucun des paramètres considérés. Ainsi, on peut comparer les concentrations de $\mathrm{NaCl}$ entre elles indépendamment des cultivars d'une part, et les cultivars entre eux indépendamment des concentrations de $\mathrm{NaCl}$ d'autre part.

Tableau 1 : Résultats de l'analyse de la variance à deux facteurs pour différents paramètres de croissance de cinq cultivars de piment (Adologbo, Gbatakin, Gbatakin d'Agbédranfo, TPS0251 et Démon) cultivés en présence de différentes concentrations de $\mathrm{NaCl}$

\begin{tabular}{|c|c|c|c|}
\hline Paramètres & Stress & Cultivars & Interaction (Stress x Cultivars) \\
\hline $\mathrm{CRH}$ & $16,98^{* \star *}$ & $4,69^{\star \star}$ & 1,53 ns \\
\hline CRLR & $2,92^{*}$ & $3,21^{*}$ & 0,31 ns \\
\hline CRNF & $4,46^{\star *}$ & $2,13^{\mathrm{ns}}$ & $0,47 \mathrm{~ns}$ \\
\hline & & & \\
\hline MSPA & $8,50^{* \star *}$ & $6,39^{* * *}$ & $0,77^{\mathrm{ns}}$ \\
\hline MSPR & $5,40^{* \star *}$ & $3,11^{*}$ & $0,56^{\mathrm{ns}}$ \\
\hline MFPR & $10,04^{\star \star \star}$ & $4,78^{*}$ & 0,72 ns \\
\hline CRSF & $11,45^{\star \star *}$ & $72,43^{* * *}$ & 1,36 ns \\
\hline
\end{tabular}

Les valeurs de F sont données pour les effets des facteurs suivants : stress (présence de $\mathrm{NaCl}$ dans le milieu), cultivar et l'interaction entre le stress et le cultivar.

**: différence hautement significative à $p=0,01^{*}$ : différence significative à $p=0,05$ ns: différence non significative

Effet du $\mathrm{NaCl}$ sur la croissance des plants : L'effet du $\mathrm{NaCl}$ se traduit par une diminution de la hauteur des plantes (Photo 1). La figure 1 indique que le rythme de diminution varie en fonction de la concentration de $\mathrm{NaCl}$ et du cultivar. Les tableaux 2 et 3 indiquent que la diminution de hauteur est significative $(p=0,001)$ à partir de $60 \mathrm{mM} \mathrm{NaCl}$ pour tous les cultivars pris ensemble et que la croissance en hauteur du cultivar Adologbo est significativement plus faible $(p=0,01)$.sous l'effet du $\mathrm{NaCl}$ que celle du cultivar Gbatakin; les trois autres cultivars présentent un comportement intermédiaire. 

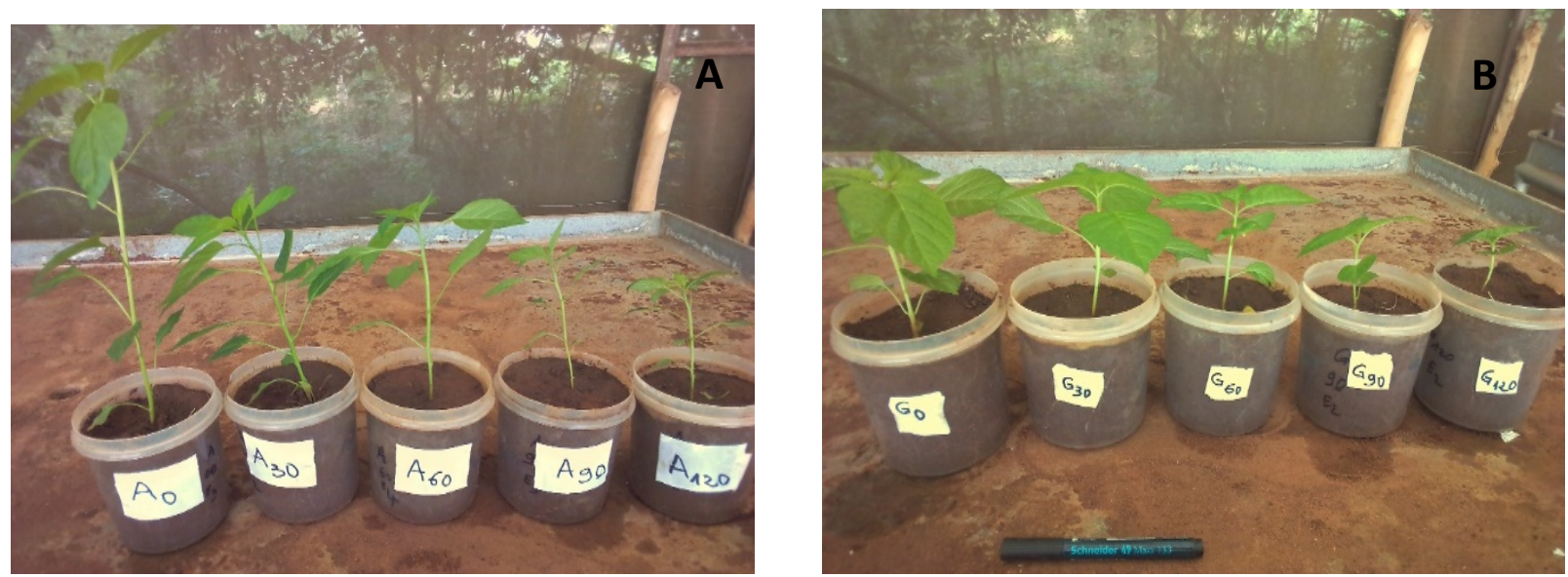

Photo 1 : Hauteur des plants de piment (A, cultivar 'Adologbo' et $B$, cultivar 'Gbatakin') après deux semaines de culture en présence de différentes concentrations de $\mathrm{NaCl}(0 ; 30 ; 60 ; 90$ et $120 \mathrm{mM}))$.

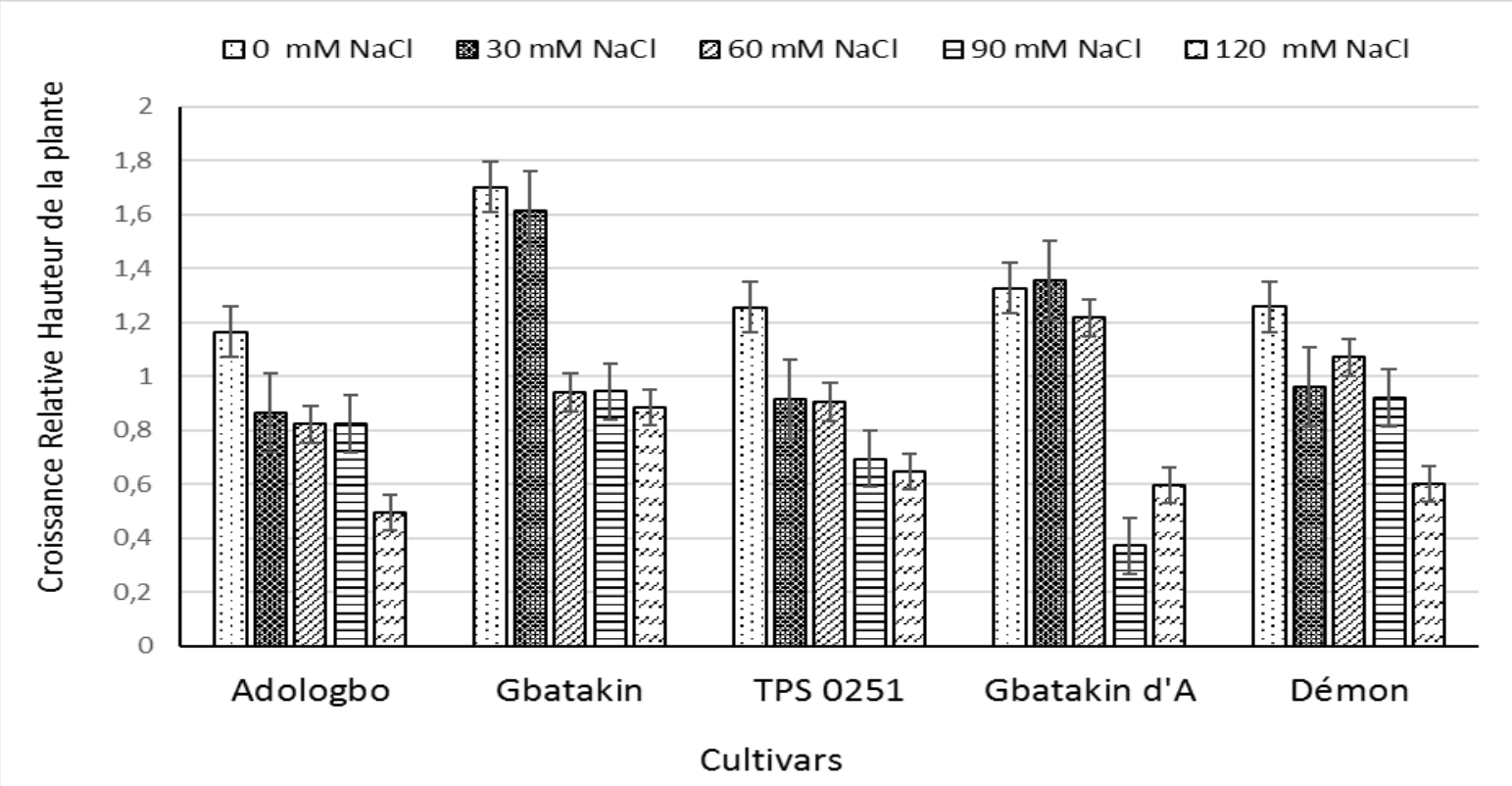

Figure 1: Croissance relative en hauteur $(\mathrm{CRH})$ des plantes de cinq cultivars de piment, cultivées pendant deux semaines en présence de différentes concentrations de $\mathrm{NaCl}(n=4$; la barre verticale correspond à l'erreur standard).

Tableau 2 : Effet de différentes concentrations de $\mathrm{NaCl}(0 ; 30 ; 60 ; 90$ et $120 \mathrm{mM})$ sur les différents paramètres de croissance chez 5 cultivars de piment.

\begin{tabular}{l|l|l|l|l|l|l|l|l}
\hline $\begin{array}{l}\text { Concentrations } \\
\text { NaCl (mM) }\end{array}$ & CRH & CRNF & CRSF & MFPA & MSPA & CRLR & MFPR & MSPR \\
\hline 0 & $1,34 \mathrm{a}$ & $1,21 \mathrm{a}$ & $126,77 \mathrm{a}$ & $5,17 \mathrm{a}$ & $4,52 \mathrm{a}$ & $1,77 \mathrm{a}$ & $5,35 \mathrm{a}$ & $4,53 \mathrm{a}$ \\
30 & $1,14 \mathrm{ab}$ & $0,88 \mathrm{ab}$ & $120,49 \mathrm{ab}$ & $3,91 \mathrm{ab}$ & $4,00 \mathrm{ab}$ & $1,49 \mathrm{ab}$ & $3,41 \mathrm{ab}$ & $4,13 \mathrm{ab}$ \\
60 & $0,99 \mathrm{bc}$ & $0,88 \mathrm{ab}$ & $111,60 \mathrm{ab}$ & $3,07 \mathrm{bc}$ & $3,24 \mathrm{abc}$ & $1,29 \mathrm{ab}$ & $2,86 \mathrm{bc}$ & $3,34 \mathrm{abc}$ \\
90 & $0,75 \mathrm{~cd}$ & $0,74 \mathrm{~b}$ & $89,77 \mathrm{ab}$ & $2,37 \mathrm{c}$ & $2,56 \mathrm{bc}$ & $1,06 \mathrm{ab}$ & $1,05 \mathrm{bc}$ & $1,86 \mathrm{bc}$ \\
120 & $0,65 \mathrm{~d}$ & $0,66 \mathrm{~b}$ & $65,25 \mathrm{~b}$ & $1,61 \mathrm{c}$ & $1,72 \mathrm{c}$ & $0,98 \mathrm{~b}$ & $0,83 \mathrm{c}$ & $1,27 \mathrm{c}$ \\
Erreur standard & 0,076 & 0,1 & 15,03 & 0,37 & 0,42 & 0,18 & 0,62 & 0,60 \\
\hline
\end{tabular}

Les moyennes avec des lettres différentes dans la même colonne sont significativement différentes à un seuil de $p=0,001$. 


\section{Kpinkoun et al., J. Appl. Biosci. 2019 Évaluation de la résistance à la salinité au stade jeune plant de quelques cultivars de piment (Capsicum spp.) du Benin}

Tableau 3 : Réponse des cinq cultivars de piment au $\mathrm{NaCl}(0 ; 30 ; 60 ; 90$ et $120 \mathrm{mM})$ au niveau de différents paramètres de croissance après deux semaines de culture

\begin{tabular}{l|l|l|l|l|l|l|l|l}
\hline Cultivars & CRH & CRNF & CRSF & MFPA & MSPA & CRLR & MFPR & MSPR \\
\hline Adologbo & $0,83 \mathrm{~b}$ & $0,92 \mathrm{a}$ & $44,91 \mathrm{c}$ & $2,05 \mathrm{c}$ & $1,97 \mathrm{c}$ & $0,75 \mathrm{~b}$ & $1,52 \mathrm{~b}$ & $2,58 \mathrm{ab}$ \\
TPS0251 & $0,88 \mathrm{ab}$ & $0,78 \mathrm{a}$ & $54,07 \mathrm{c}$ & $2,55 \mathrm{bc}$ & $2,88 \mathrm{~b}$ & $1,31 \mathrm{ab}$ & $1,74 \mathrm{ab}$ & $1,88 \mathrm{~b}$ \\
Gbatakin & $1,22 \mathrm{a}$ & $0,86 \mathrm{a}$ & $204,53 \mathrm{a}$ & $4,33 \mathrm{a}$ & $4,64 \mathrm{a}$ & $1,55 \mathrm{a}$ & $4,31 \mathrm{a}$ & $4,59 \mathrm{a}$ \\
Gbatakin & $0,99 \mathrm{ab}$ & $0,71 \mathrm{a}$ & $104,59 \mathrm{~b}$ & $4,02 \mathrm{ab}$ & $3,20 \mathrm{ab}$ & $1,47 \mathrm{ab}$ & $4,02 \mathrm{ab}$ & $3,68 \mathrm{ab}$ \\
d'Agbédranfo & & & & & & & & \\
Démon & $0,96 \mathrm{ab}$ & $1,09 \mathrm{a}$ & $105,79 \mathrm{~b}$ & $3,31 \mathrm{abc}$ & $3,44 \mathrm{ab}$ & $1,54 \mathrm{a}$ & $2,05 \mathrm{ab}$ & $2,53 \mathrm{ab}$ \\
Erreur standard & 0,09 & 0,10 & 9,13 & 0,42 & 0,43 & 0,18 & 0,67 & 0,63 \\
\hline
\end{tabular}

Les moyennes avec des lettres différentes dans la même colonne sont significativement différentes à un seuil de $p=0,001$.

L'effet du $\mathrm{NaCl}$ se traduit par une diminution du nombre de feuilles des plantes au niveau des cinq cultivars (Figure 2). Les tableaux 2 et 3 indiquent que la diminution du nombre de feuilles est significative $(p=$ $0,01)$ à partir de $90 \mathrm{mM} \mathrm{NaCl}$ et que les cinq cultivars présentent à peu près les mêmes comportements.

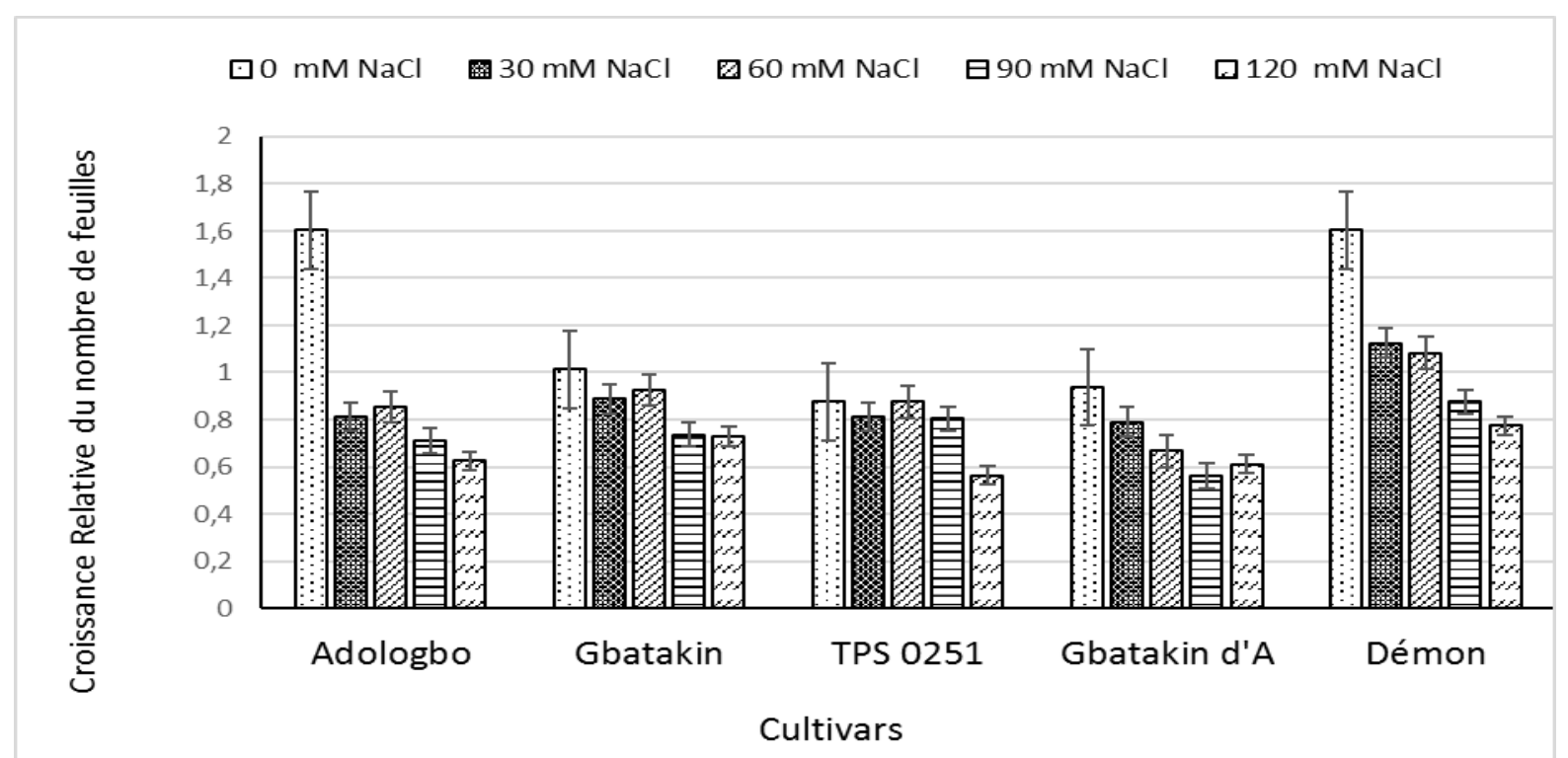

Figure 2: Croissance relative du nombre de feuilles (CRNF) des plantes de cinq cultivars de piment, cultivées pendant deux semaines en présence de différentes concentrations de $\mathrm{NaCl}(n=4$; la barre verticale correspond à l'erreur standard).

L'effet du $\mathrm{NaCl}$ se traduit par une diminution de la surface foliaire des plantes et le rythme de diminution varie en fonction de la concentration de $\mathrm{NaCl}$ et du cultivar (Figure 3). Les tableaux 2 et 3 indiquent que la diminution de croissance est significative $(p=0,001)$ uniquement à $120 \mathrm{mM} \mathrm{NaCl}$ et que le cultivar Gbatakin maintient une surface foliaire significativement plus élevée $(p=0,001)$ en présence du $\mathrm{NaCl}$ que les quatre autres cultivars; les cultivars TPS0251 et Adologbo présentent les surfaces foliaires les plus faibles. 


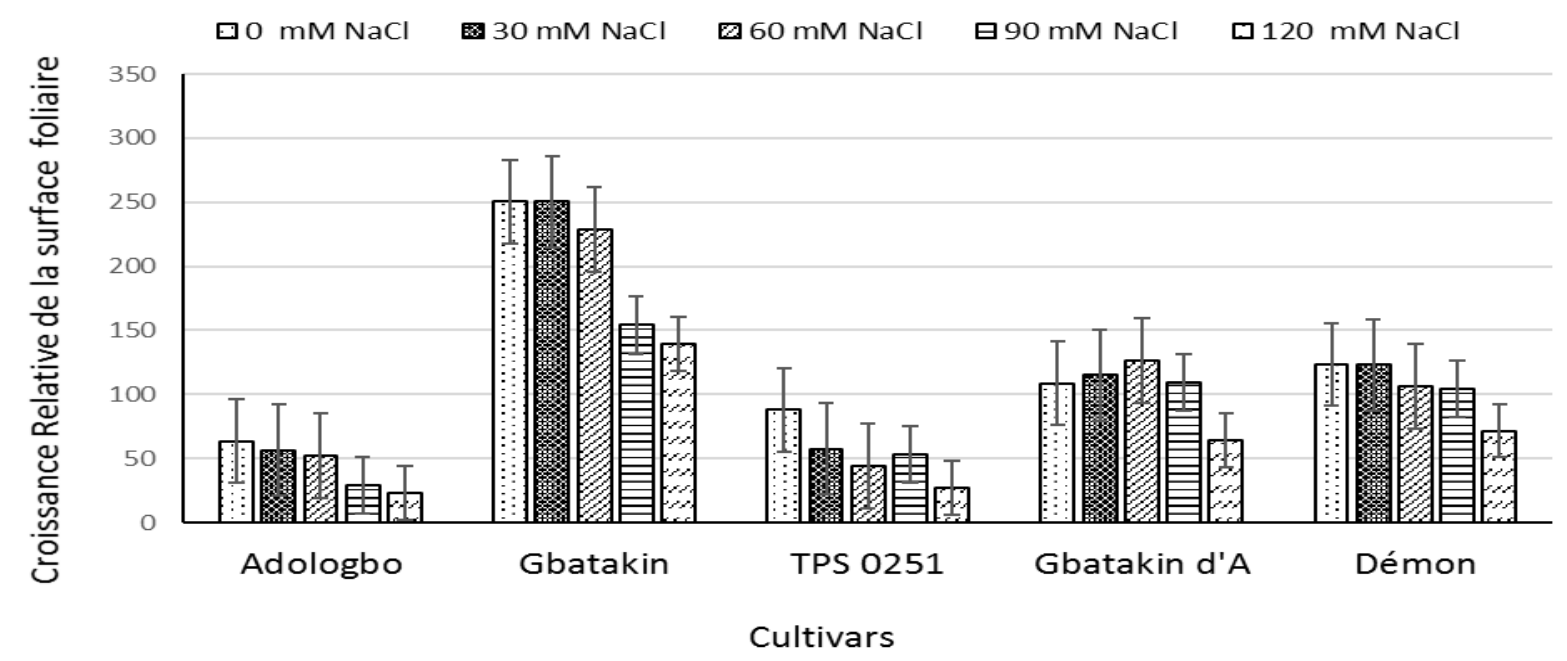

Figure 3: Croissance relative de la surface foliaire (CRSF) des plantes de cinq cultivars de piment, cultivées pendant deux semaines en présence de différentes concentrations de $\mathrm{NaCl}(n=4$; la barre verticale correspond à l'erreur standard).

L'effet du $\mathrm{NaCl}$ se traduit par une diminution de la masse fraîche de la partie aérienne des plantes et le rythme de diminution varie en fonction de la concentration de $\mathrm{NaCl}$ et du cultivar (Figure 4). Les tableaux 2 et 3 indiquent que la diminution de la masse fraîche est significative $(p=0,001)$ à partir de $60 \mathrm{mM}$ $\mathrm{NaCl}$ et que le cultivar Gbatakin maintient la masse fraîche de la partie aérienne significativement plus élevée $(p=0,001)$ en présence du $\mathrm{NaCl}$ que les cultivars TPS0251 et Adologbo ; les deux autres cultivars sont intermédiaires. L'effet du $\mathrm{NaCl}$ se traduit par une diminution de la masse sèche de la partie aérienne des plantes et le rythme de diminution varie en fonction de la concentration de $\mathrm{NaCl}$ et du cultivar (Figure 5). Une légère stimulation est observée à $30 \mathrm{mM} \mathrm{NaCl}$ pour le cultivar Gbatakin. Les tableaux 2 et 3 indiquent que la diminution de croissance est significative $(p=0,001)$ à partir de $90 \mathrm{mM} \mathrm{NaCl}$ et que le cultivar Gbatakin maintient la masse sèche de la partie aérienne significativement plus élevée $(p=0,001)$ en présence $d u$ $\mathrm{NaCl}$ que les cultivars TPS0251 et Adologbo ; les deux autres cultivars sont intermédiaires.

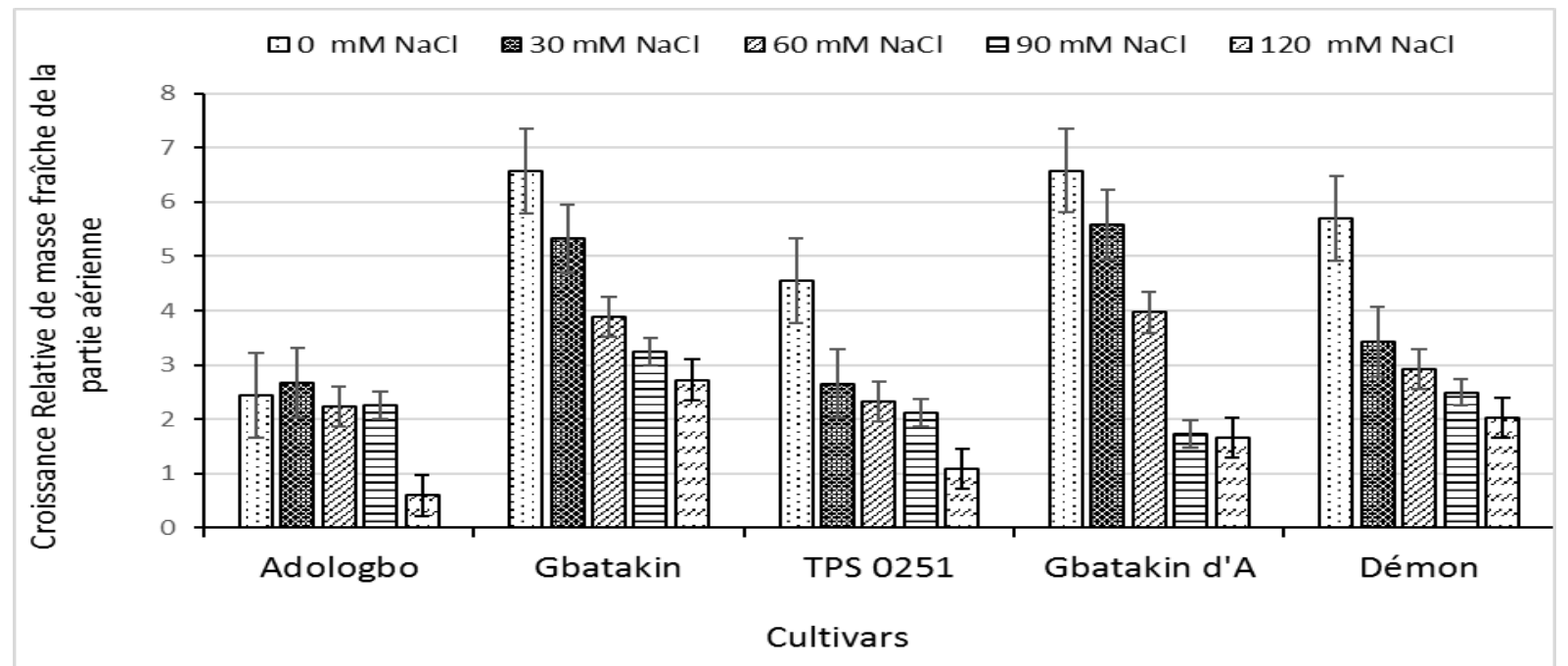

Figure 4 : Croissance relative de la masse fraîche de la partie aérienne (CRMFPA) des plantes de cinq cultivars de piment, cultivées pendant deux semaines en présence de différentes concentrations de $\mathrm{NaCl}(\mathrm{n}=4$; la barre verticale correspond à l'erreur standard). 


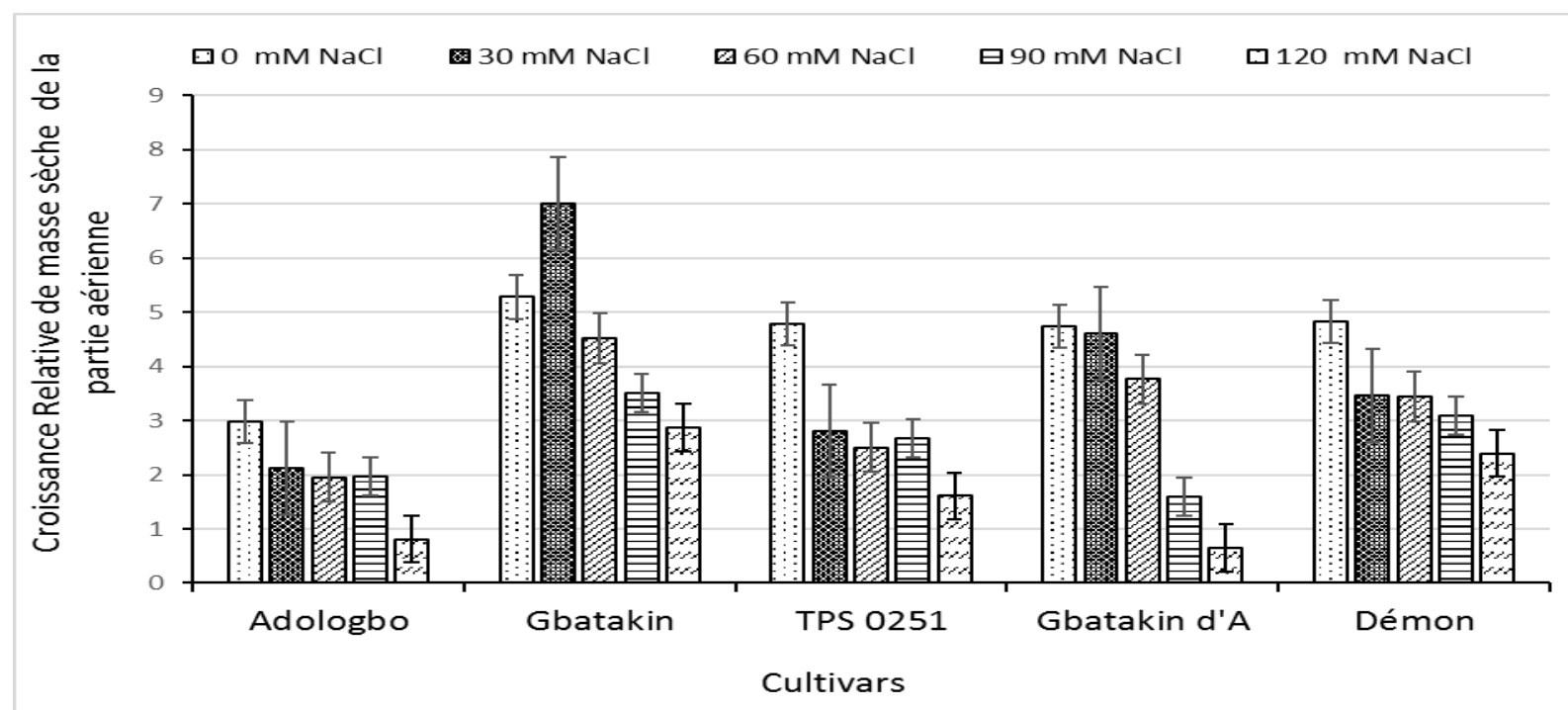

Figure 5 : Croissance relative de la masse sèche de la partie aérienne (CRMSPA) des plantes de cinq cultivars de piment, cultivées pendant deux semaines en présence de différentes concentrations de $\mathrm{NaCl}(n=4$; la barre verticale correspond à l'erreur standard).

L'effet du $\mathrm{NaCl}$ se traduit par une diminution de la croissance en longueur des racines au fur et à mesure que la concentration de $\mathrm{NaCl}$ augmente dans le milieu de culture et le rythme de diminution varie en fonction du cultivar (Figure 6). Les tableaux 2 et 3 indiquent que la diminution de croissance est significative $(p=0,05)$ uniquement à $120 \mathrm{mM} \mathrm{NaCl}$ et que la longueur des racines du cultivar Adologbo est significativement plus réduite en présence de $\mathrm{NaCl}$ que celle des autres cultivars $(p=0,05)$.

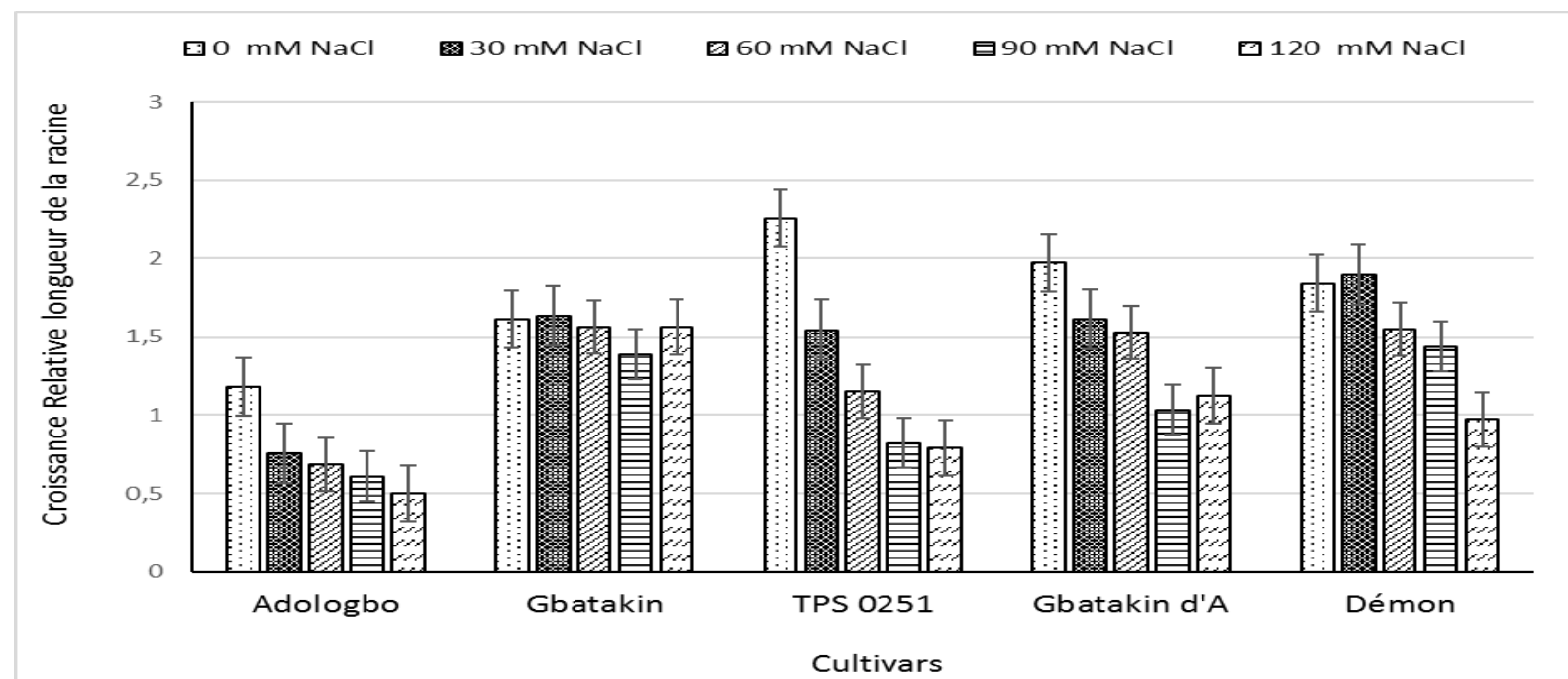

Figure 6 : Croissance relative de la longueur des racines (CRLR) des plantes de cinq cultivars de piment, cultivées pendant deux semaines en présence de différentes concentrations de $\mathrm{NaCl}(\mathrm{n}=4$; la barre verticale correspond à l'erreur standard).

L'effet du $\mathrm{NaCl}$ se traduit par une diminution de la masse fraîche des racines et le rythme de diminution varie en fonction de la concentration de $\mathrm{NaCl}$ et du cultivar (Figure 7). Les tableaux 2 et 3 indiquent que la diminution de croissance est significative $(p=0,001)$ à partir de $60 \mathrm{mM} \mathrm{NaCl}$ et que la masse fraîche des racines du cultivar Adologbo est significativement plus réduite en présence de $\mathrm{NaCl}$ que celle des autres cultivars $(p=0,05)$. 


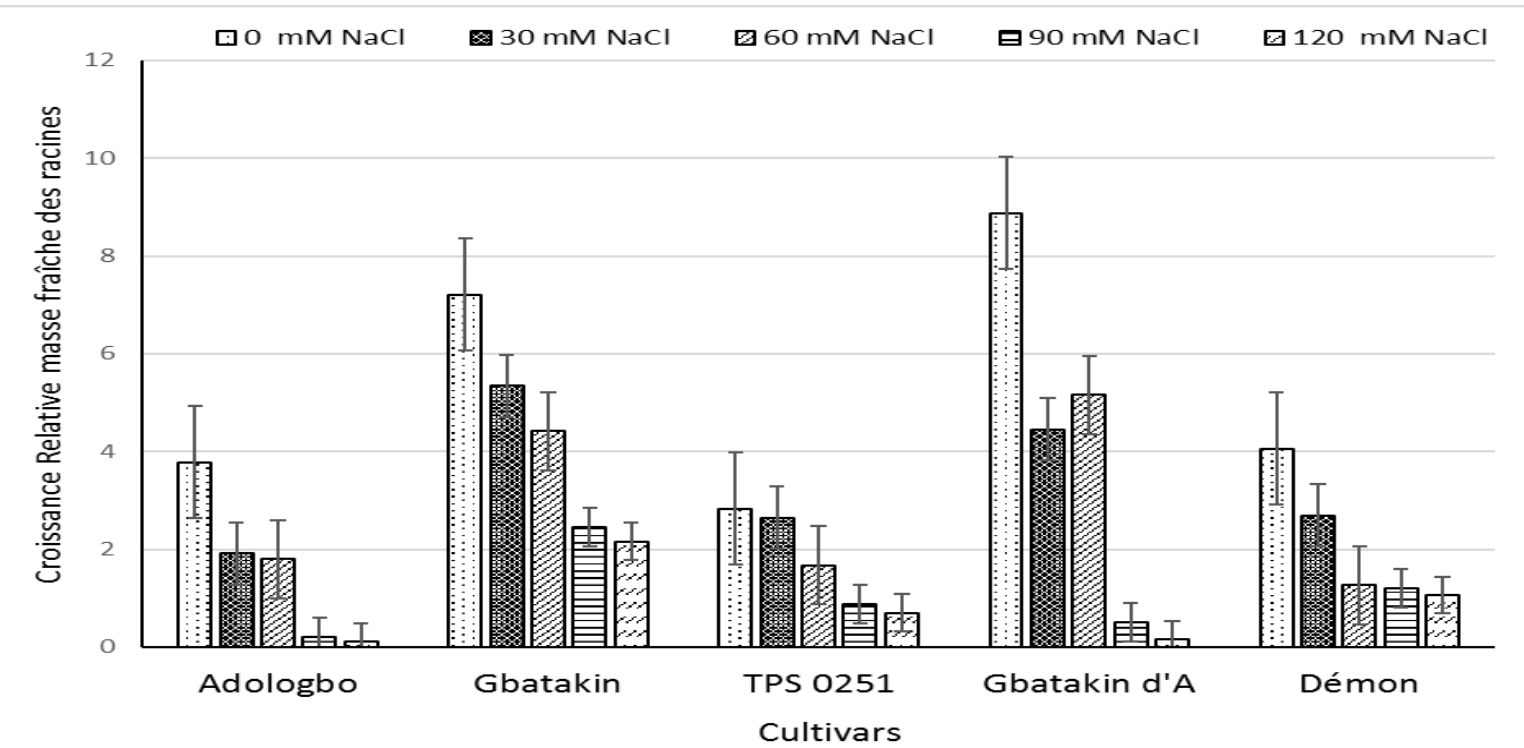

Figure 7 : Croissance relative de la masse sèche des racines (CRMSR) des plantes de cinq cultivars de piment, cultivées pendant deux semaines en présence de différentes concentrations de $\mathrm{NaCl}(n=4$; la barre verticale correspond à l'erreur standard).

L'effet du $\mathrm{NaCl}$ se traduit par une diminution de la masse sèche des racines et le rythme de diminution varie en fonction de la concentration de $\mathrm{NaCl}$ et du cultivar (Figure 8). Une légère stimulation est observée à $60 \mathrm{mM}$ de $\mathrm{NaCl}$ pour les cultivars Adologbo et Gbatakin d'Agbédranfo. Les tableaux 2 et 3 indiquent que la diminution de croissance est significative $(p=0,001)$ à partir de $90 \mathrm{mM} \mathrm{NaCl}$ et que la masse sèche des racines du cultivar TPS 0251 est significativement plus réduite $(p=0,05)$.en présence de $\mathrm{NaCl}$ que celle des autres cultivars.

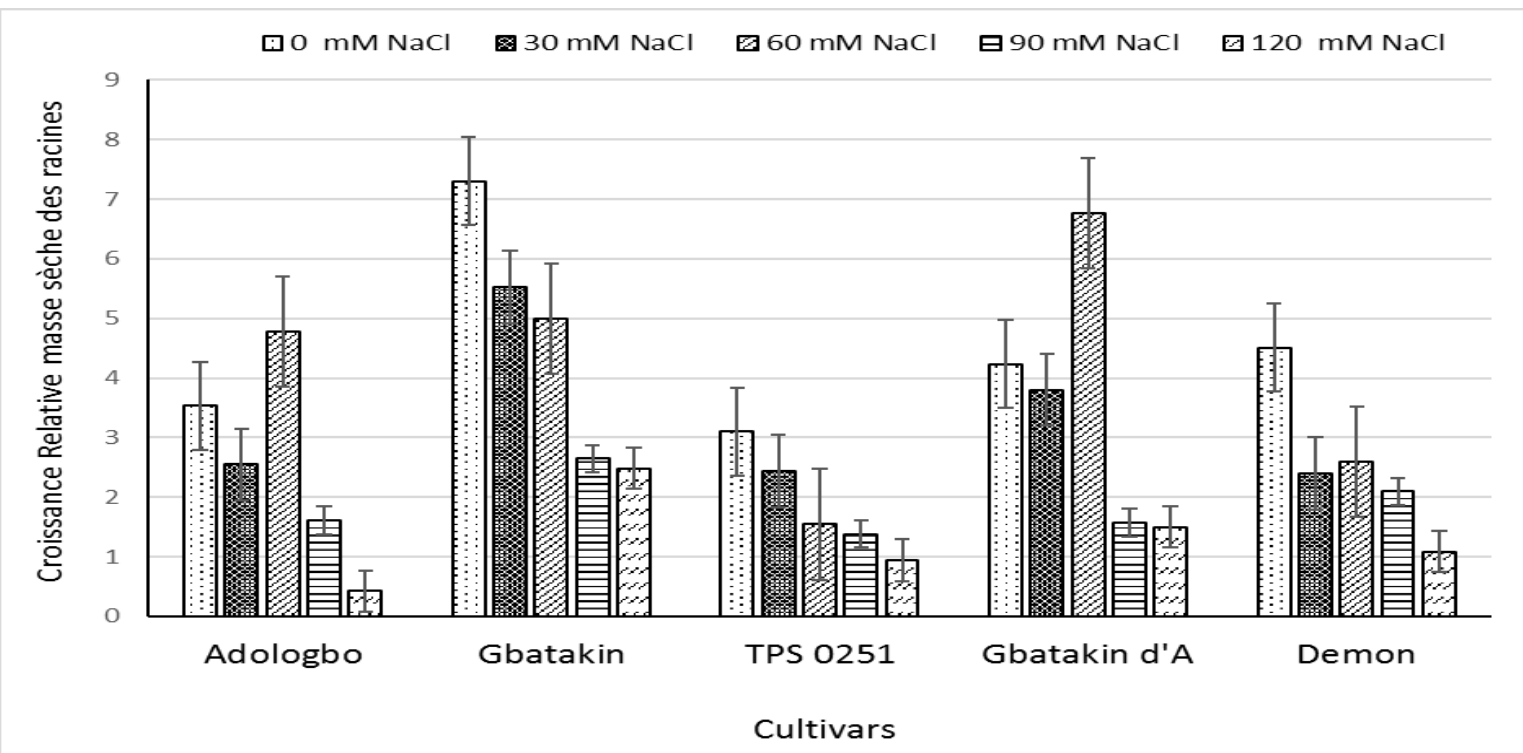

Figure 8 : Croissance relative de la masse sèche des racines (CRMSR) des plantes de cinq cultivars de piment, cultivées pendant deux semaines en présence de différentes concentrations de $\mathrm{NaCl}(n=4$; la barre verticale correspond à l'erreur standard). 
En considérant les trois paramètres évalués en même temps au niveau de la partie aérienne et de la partie racinaire à savoir : hauteur/longueur, masse de matière fraîche et masse de matière sèche), on peut observer que l'effet du stress est significatif à partir des mêmes concentrations de $\mathrm{NaCl}$ pour la masse de matière fraîche $(60 \mathrm{mM})$ et la masse de matière sèche $(90 \mathrm{mM})$ pour les deux parties de la plante. Par contre pour la hauteur de la plante, l'effet du $\mathrm{NaCl}$ est significatif à partir de $60 \mathrm{mM}$ alors qu'il n'est significatif pour la longueur de la racine qu'à seulement $120 \mathrm{mM}$. Ces résultats suggèrent que la partie aérienne est plus affectée par le stress salin que la partie racinaire. Indice de tolérance au sel (ITS) des cultivars: Le tableau 4 présente l'indice de tolérance au sel des cinq cultivars pour les différents paramètres étudiés. L'analyse de variance à un facteur révèle une différence significative entre les cultivars en ce qui concerne leur indice de tolérance au sel pour l'ensemble des paramètres de croissance pris en compte. Excepté pour le nombre de feuilles, le cultivar Gbatakin présente l'indice le plus élevé alors que les cultivars TPS 0251 et Adologbo présentent les indices les plus faibles; les autres cultivars présentent des indices intermédiaires.

Tableau 4: Indice de tolérance au sel (ITS) de cinq cultivars de piment ('Adologbo'; 'Gbatakin'; 'TPS 0251'; 'Gbatakin d'Agbodranfo' et 'Démon') pour différents paramètres de croissance en présence de cinq concentrations de $\mathrm{NaCl}(0 ; 30 ; 60.90$ et $120 \mathrm{mM})$

\begin{tabular}{l|l|l|l|l|l}
\hline variétés & Gbatakin & $\begin{array}{l}\text { Gbatakin } \\
\text { d'Agbédranfo }\end{array}$ & Démon & TPS 0251 & Adologbo \\
\hline CRHPA & $1,052 \pm 0,121^{\mathrm{a}}$ & $0,670 \pm 0,123^{\mathrm{b}}$ & $0,613 \pm 0,050^{\mathrm{b}}$ & $0,555 \pm 0,072^{\mathrm{b}}$ & $0,474 \pm 0,043^{\mathrm{b}}$ \\
CRMFPA & $0,796 \pm 0,096^{\mathrm{a}}$ & $0,780 \pm 0,152^{\mathrm{a}}$ & $0,606 \pm 0,095^{\mathrm{ab}}$ & $0,314 \pm 0,056^{\mathrm{bc}}$ & $0,161 \pm 0,029^{\mathrm{c}}$ \\
CRMSPA & $1,035 \pm 0,203^{\mathrm{a}}$ & $0,582 \pm 0,115^{\mathrm{abc}}$ & $0,722 \pm 0,118^{\mathrm{abc}}$ & $0,491 \pm 0,078^{\mathrm{bc}}$ & $0,217 \pm 0,041^{\mathrm{c}}$ \\
CRSF & $2,883 \pm 0,27^{\mathrm{a}}$ & $0,685 \pm 0,08^{\mathrm{bc}}$ & $0,757 \pm 0,05^{\mathrm{bc}}$ & $0,257 \pm 0,04^{\mathrm{cd}}$ & $0,159 \pm 0,02^{\mathrm{d}}$ \\
CRNF & $0,865 \pm 0,162^{\mathrm{a}}$ & $0,652 \pm 0,111^{\mathrm{a}}$ & $1,549 \pm 0,140^{\mathrm{a}}$ & $0,633 \pm 0,102^{\mathrm{a}}$ & $1,794 \pm 0,683^{\mathrm{a}}$ \\
CRLR & $0,770 \pm 0,115^{\mathrm{ab}}$ & $0,639 \pm 0,107^{\mathrm{ab}}$ & $0,888 \pm 0,178^{\mathrm{a}}$ & $0,314 \pm 0,056^{\mathrm{bc}}$ & $0,161 \pm 0,029^{\mathrm{c}}$ \\
CRMFPR & $1,011 \pm 0,222^{\mathrm{a}}$ & $0,684 \pm 0,343^{\mathrm{ab}}$ & $0,304 \pm 0,095^{\mathrm{ab}}$ & $0,0908 \pm 0,027^{\mathrm{b}}$ & $0,145 \pm 0,081 \mathrm{~b}$ \\
CRMSPR & $1,286 \pm 0,297^{\mathrm{a}}$ & $0,554 \pm 0,107^{\mathrm{b}}$ & $0,468 \pm 0,087^{\mathrm{b}}$ & $0,238 \pm 0,072^{\mathrm{b}}$ & $0,333 \pm 0,106^{\mathrm{b}}$ \\
\hline
\end{tabular}

Les moyennes avec des lettres différentes sur la même ligne sont significativement différentes à un seuil de $p=0,05$.

\section{DISCUSSION}

Le stress salin a provoqué une inhibition évidente de la croissance de la partie aérienne et des racines chez tous les cultivars au stade jeune plant. Cette observation est communément signalée pour les glycophytes comme le blé (Alaoui et al., 2013), le riz (Hussain et al., 2017), la canne à sucre (Gandonou et al., 2012 ; Gandonou \& Skali Senhaji, 2015), la tomate (Albacete et al., 2008; Ould Mohamdi et al., 2011), l'amarante (Omami, 2005 ; Omami \& Hammes, 2006 ; Wouyou et al., 2017). Des résultats similaires ont également été rapportés pour l'orge et le maïs par Cramer (2003) qui a constaté que le stress salin réduit l'allongement de la partie aérienne. Chez le piment, R'him et al. (2013) ont signalé que le stress salin a réduit la croissance de cinq génotypes en utilisant différents critères de croissance tels que la hauteur de la plante, le nombre de feuilles, la surface foliaire, la masse foliaire et la masse fraîche et sèche. La même tendance a été observée chez d'autres génotypes de piment (Chartzoulakis \& Klapaki, 2000 ; Rubio et al.,
2010). La réduction de la croissance des plantes, en particulier dans l'accumulation de la biomasse, pourrait être la conséquence d'un stress hydrique résultant d'une diminution du potentiel osmotique externe et/ou de l'accumulation d'ions devenant toxiques (Odjegba \& Chukwunwike, 2012). D'autres recherches sont nécessaires pour déterminer quelle composante du stress salin est la plus nocive pour Capsicum annuum. Les résultats de cette étude ont révélé un effet significatif des concentrations de $\mathrm{NaCl}$ pour tous les paramètres de croissance considérés dans cette étude, ce qui indique que la réponse des cultivars de piment au stress salin dépend de la concentration. Par ailleurs, Les résultats de cette étude ont montré que la partie aérienne est plus affectée que la partie racinaire. Des résultats similaires ont été rapportés chez les espèces du genre Phaseolus (Bayuelo-Jimenez et al., 2002) et chez l'amarante (Gandonou et al., 2018). Ces résultats peuvent s'expliquer par une aptitude plus élevée à l'ajustement osmotique sous stress salin par les racines 

quelques cultivars de piment (Capsicum spp.) du Benin

tel que rapporté chez la Vitis vinifera en particulier aux concentrations élevées de $\mathrm{NaCl}$ (Fisarakis et al., 2001). La réponse des cultivars dépend du paramètre de croissance considéré. Les résultats ont révélé que la hauteur des plantes, la masse de matière fraîche de la partie aérienne et de la partie racinaire sont les seuls paramètres significativement affectés à partir de $60 \mathrm{mM}$ $\mathrm{NaCl}$ pour l'ensemble des cultivars; les autres paramètres étant affectés à partir de $90 \mathrm{mM}$ de $\mathrm{NaCl}$. La hauteur des plantes, la masse de matière fraîche de la partie aérienne et de la partie racinaire apparaissent donc comme les paramètres les plus sensibles au stress salin chez les cultivars de piment utilisés, suivi du nombre de feuilles, de la masse sèche de la partie aérienne et de la masse sèche de la partie racinaire. La surface foliaire et la croissance en longueur de la racine ne sont affectées qu'à la plus grande concentration de $\mathrm{NaCl}$ utilisée (120 mM). Ainsi, la hauteur de la plante, la masse de matière fraîche de la partie aérienne et de la partie racinaire apparaissent comme les paramètres de croissance les plus appropriés pour l'étude de l'effet du stress salin chez le piment. II est largement admis que le stress salin agit sur la croissance des plantes de trois manières : à travers les effets osmotiques, à travers la toxicité spécifique des ions salins et/ou à travers les perturbations nutritionnelles. Selon Läuchli \& Epstein (1990), ces trois facteurs ont un effet direct sur la hauteur de la plante. On peut donc supposer que les

\section{CONCLUSION}

Cette étude a montré que le stress salin réduit la croissance des jeunes plants chez les cultivars de piment notamment à partir d'une concentration de 60 $\mathrm{mM}$ de $\mathrm{NaCl}$. Elle a révélé la variabilité de la résistance relative au stress salin pour des cultivars locaux de piment au stade de jeunes plants. La hauteur de la plante, la masse de matière fraîche de la partie aérienne et de la partie racinaire apparaissent comme les paramètres de croissance les plus appropriés pour réductions de croissance observées chez les différents cultivars de piment testés sont dues à l'un ou l'autre ou encore à la combinaison de deux ou de ces trois facteurs. II est bien connu que, pour les espèces végétales, il existe une variation substantielle de la sensibilité au sel chez les cultivars de la même espèce (Lutts et al., 1995; Wei et al., 2003; Gandonou et al., 2012) .Les résultats de cette étude confirment que c'est également le cas pour Capsicum spp. car une différence significative a été observée parmi les cultivars testés. Le cultivar Gbatakin présente le meilleur comportement vis-à-vis du sel avec l'Indice de Tolérance au Sel le plus élevé quel que soit le paramètre de croissance pris en compte (excepté le nombre de feuilles); de ce fait, il apparaît comme le cultivar le plus résistant à la salinité parmi les 5 cultivars testés. A l'inverse, les cultivars TPS 0251 et Adologbo présentent les pires comportements vis-à-vis du sel avec des Indices de Tolérance au Sel les plus faibles pour la majorité des paramètres de croissance pris en compte ; ils apparaissent de ce fait comme les cultivars les plus sensibles au stress salin. Les deux autres cultivars Gbatakin d'Agbédranfo et Démon présentent un comportement intermédiaire. Ainsi, il existe une variabilité de la résistance relative au stress salin chez les cultivars de Capsicum spp. au stade de jeunes plants et en particulier chez les cultivars locaux du Bénin.

l'étude de l'effet du stress salin chez le piment. Parmi les cinq cultivars testés et quel que soit le paramètre de croissance pris en compte, le cultivar Gbatakin est le plus résistant tandis que les cultivars Adologbo et TPS 0251 sont les plus sensibles. A ce stade des travaux, le cultivar Gbatakin peut être conseillé aux producteurs des zones côtières ou affectées par la salinité tandis que les producteurs des autres zones peuvent produire tous les cultivars.

\section{REMERCIEMENTS}

Les auteurs remercient le Professeur Alexandre Dansi Anagonou de l'Université d'Abomey-Calavi pour les corrections apportées au manuscrit.

\section{REFERENCES}

Akinci IE, Akinci S, Yilmaz, K, Dikici, H, 2004. Response of eggplant varieties (Solanum melongena) to salinity in germination and seedling stages. New Zealand Journal of Crop Horticultural Science 32: 193-200.

Akram M, Hussain M, Akhtar, S, Rasul E, 2002. Impact of $\mathrm{NaCl}$ salinity on yield components of some wheat accession/ variety. International Journal of Agriculture and Biology 4: 156-158. 
Alaoui MM, El Jourmi L, Ouarzane A, Lazar S, El Antri S, Zahouily M, Hmyene A, 2013. Effet du stress salin sur la germination et la croissance de six variétés marocaines de blé. J. Mater. Environ. Sci. 4 (6) :997-1004

Albacete A, Ghanem ME, Martinez C, Jari, A, Acosta M, Sanchez-Bravo J, 2008. Hormonal changes in relation to biomass partitioning and shoot growth impairment in salinized tomato (Solanum lycopersicum L.) plants. Journal of Experimental Botany 59 (15): 4119-4131.

Assogba-Komlan F, Sodjinou E, Mensah A, Sikirou R, 2009. Production durable du piment au Bénin. Institut National des Recherches Agricoles du Bénin, $48 \mathrm{p}$.

Bayuelo-Jiménez JS, Graig R, Lynch JP, 2002. Salinity tolerance of Phaseolus species during early vegetative growth. Crop Science 42: 21842192.

Bhattacharjee S, 2008. Triadimef on pretreatment protects newly assembled membrane system and causes up-regulation of stress proteins in salinity stressed Amaranthus lividus L. during early germination. Journal of Environmental Biology 29 (5): 805- 810.

Caravajal M, Amor FM, Fernandez-Ballester G, Martinez V, Cerdia A, 1998. Time course of solute accumulation and water relations in muskmelon plants exposed to salt during different growth stages. Plant Science 138: 103-112.

Chartzoulakis K, Klapaki G, 2000. Response of two greenhouse pepper hybrids to $\mathrm{NaCl}$ salinity during different growth stages. Scientia Horticulturae 86: 247-260.

Chukwu LO, Okpe HA, 2006. Differential response of Tilapia guineensis fingerlings to inorganic fertilizer under various salinity regimes. Journal of Environmental Biology 27: 687-690.

Cramer GR, 2003. Differential effects of salinity on leaf elongation kinetics of three grass species. Plant and Soil 253 : 233-244.

Déguénon E. 2018. Communication présentée le mercredi 30 mai 2018 lors du Forum des jeunes chercheurs sur la Physiologie Végétale et ses applications au service du développement de l'agriculture: Cas de l'igname et des cultures maraîchères.

Fisarakis I, Chartzoulakis K, Stavrakas D, 2001. Response of sultana vines ( $V$. vinifera $L$.) on six rootstocks to $\mathrm{NaCl}$ salinity exposure and recovery. Agriculture Water Manage 51:13-27.

Gandonou CB, Gnancadja SL, Abrini J, Skali Senhaji N, 2012. Salinity tolerance of some sugarcane (Saccharum sp.) cultivars in hydroponic medium. International Sugar Journal 114 (1359): 190-196.

Gandonou CB, Skali Senhaji N, 2015. Sugarcane (Saccharum sp.) salt tolerance at various developmental levels. In: Chakraborty U, Chakraborty B, Editors. Abiotic Stresses in Crop Plants, $\mathrm{CABI}$ Publishing, United Kingdom, ISBN-13: 978-1-78064-373-1

Gandonou CB, Prodjinoto H, Zanklan SA, Wouyou AD, Lutts S, Montcho DH, Assogba Komlan F, Mensah ACG, 2018. Effects of salinity stress on growth in relation to gas exchanges parameters and water status in amaranth (Amaranthus cruentus). International Journal of Plant Physiology and Biochemistry 10(3): 19-27.

Hussain K, Majeed A, Nawaz K, Khizar HB, Nisar MF, 2009. Effect of different levels of salinity on growth and ion contents of black seeds (Nigella sativa L.). Current Research Journal of Biological Sciences 1: 135-138.

Hussain S, Zhang J-H, Zhong C, Zhu L-F, Cao X-C, Yu, S-M, Hu JABJ-J, Jin Q-J, 2017. Effects of salt stress on rice growth, development characteristics, and the regulating ways: $A$ review. Journal of Integrative Agriculture 16 (11): 2357-2374.

Karikalan L, Rajan SN, Gopi R, Sujatha BM, Pannersevalam $R$, 1999. Induction of salt tolerance by triadimefon in pigeon pea (Cajanus cajan L.) Mill sp. Indian Journal of Experimental Biology 37:825-829.

Lakra N, Mishra SN, Singh DB, Pushpa CT, 2006. Exogenous putrescine effect on cation concentration in leaf of Brassica juncea seedlings subjected to $\mathrm{Cd}$ and $\mathrm{Pb}$ along with salinity stress. Journal of Environmental Biology 27: 263-269.

Läuchli A, Epstein E, 1990. Plant responses to saline and sodic conditions. Agricultural Salinity Assessment and Management 71:113-137.

Levitt J, 1980. Responses of plants to environment stresses: water, radiation, salt and other stresses, vol. 2, Acad press, New York, 520 p.

Lutts S, Guerrier G, 1995. Peroxydase activities of two rice cultivars differing in salinity tolerance as 
affected by proline and $\mathrm{NaCl}$. Biologia Plantarum 37 (4): 577-586.

Munns R, 2002. Comparative physiology of salt and water stress. Plant Cell Environment 25: 239250.

Munns R, 2005. Genes and salt tolerance: Bringing them together. Plant Physiology 167: 645-663.

Munns R, Tester M, 2008. Mechanisms of salinity tolerance. Annual Review of Plant Biology 59: 651-681.

Odjegba VJ, Chukwunwike IC, 2012. Physiological responses of Amaranthus hybridus L. under salinity stress. Indian Journal of Innovations and Developments 1 (10): 742-748.

Omami EN, 2005. Response of Amaranth to salinity stress, Ph. D Thesis, University of Pretoria, South Africa. $235 \mathrm{p}$.

Omami EN, Hammes PS, Robbertse PJ, 2006. Differences in salinity tolerance for growth and water-use efficiency in some amaranth (Amaranthus spp.) genotypes. New Zealand Journal of Crop Horticultural Science 34 (1): 11-22.

Orobiyi A, Dansi A, Assogba P, Loko LY, Dansi M, Vodouhè $R$, Akouègninou $A$, Sanni $A, 2013$. Chili (Capsicum annuum L.) in southern Benin: production constraints, varietal diversity, preference criteria and participatory evaluation, International Research Journal of Agricultural Science and Soil Science, 3(4): 107-120.

Ould Mohamdi M, Bouya D, Ould Mohamed Salem A, 2011. Etude de l'effet du stress salin $(\mathrm{NaCl})$ chez deux variétés de tomate (Campbell 33 et Mongal). International Journal of Biological and Chemical Sciences 5(3): 860-900.

PNUD (Programme des Nations Unies pour le Développement), 2015. Renforcement des capacités des maraîchers des communes de Adjohoun, de Bopa, et de Ouaké sur l'amélioration des techniques de production et de réduction des impacts des risques climatiques sur la culture du piment. $64 \mathrm{p}$. Dépôt légal $n^{\circ} 7752$ du 03/02/2015 1er trimestre, Bibliothèque Nationale du Bénin

PSRSA, 2011. Plan Stratégique de Relance du Secteur Agricole, $107 p$

R'him T, Tlili I, Hnan I, llahy R, Benali A, Jebari H, 2013. Effet du stress salin sur le comportement physiologique et métabolique de trois variétés de piment (Capsicum annuum L.), Journal of Applied BioSciences 66 : 5060 5069.

Rubio JS, Rubio F, Martínez V, García-Sánchez. F, 2010. Amelioration of salt stress by irrigation management in pepper plants grown in coconut coir dust. Agricultural Water Management 97:1695-1702.

SAS Institute, 2015. SAS/STAT user's guide, Vol. 1; Release 6.03, ed. SAS Institute Inc. Cary, NC. USA.

Tabatabaei SA, Ranjbar GH, Anagholi A, 2012. Evaluation of physiological indices of salinity tolerance in forage Sorghum (Sorghum bicolor) lines, International Research Journal of Applied and Basic Sciences. 3(2) : 305-308.

Wei W, Bilsborrow EP, Hooley P, Fincham AD, Lombi E, Forster PB, 2003. Salinity induced differences in growth, ion distribution and partitioning in barley between the cultivar Maythorpe and its derived mutant Golden Promise. Plant and Soil 250: 183-191.

Wouyou A, Gandonou CB, Assogba Komlan F, Montcho D, Zanklan SA, Lutts S, Gnancadja SL, 2017. Salinity Resistance of Five Amaranth (Amaranthus cruentus) Cultivars at Young Plants Stage, International Journal of Plant and Soil Science 14(3): 1-13. 